\title{
Bioanalytical verification of sulfur mustard exposure in a Syrian family
}

\author{
Sermet Sezigen ${ }^{1}$, Ruşen Koray Eyison ${ }^{1}$, Levent Kenar ${ }^{1}$ \\ (1) University of Health Sciences, Department of Medical CBRN Defense, Ankara, Turkey
}

Date submitted:

Feb 10, 2019

Date accepted:

Apr 04, 2019

Online publication date:

June 15, 2019

\section{Corresponding Author: \\ Sermet Sezigen \\ University of Health Sciences, \\ Department of Medical CBRN \\ Defense, Ankara, Turkey \\ sermet.sezigen@sbu.edu.tr}

Keywords: Sulfur mustard, Mustard gas, Beta lyase metabolites, SBMTE, Chemical Terrorism, CBRN.

\begin{abstract}
Aims:Sulfur mustard (SM) is a well-known chemical warfare agent. It is a highly reactive compound which has cytotoxic and carcinogenic. 1,1'-sulfonylbis[2-(methylthio)ethane] (SBMTE) is a B-lyase metabolite of SM and it is the unequivocal diagnostic biomarker for SM exposure. Our study aimed to confirm SM exposure by detecting quantitative levels of SBMTE in urine samples of a family of four.

Methods:A family of four who was exposed to SM was included into the study. Urine samples of victims were analysed by using a gas chromatography-tandem mass spectrometry (GC-MSMS) method for the detection of urinary SBMTE levels. External calibration standards were used in the multiple reaction monitoring mode of GC-MS-MS.

Results:The limit of detection (LOD) for SBMTE was $1 \mathrm{ng} / \mathrm{mL}$ and the retention time for SBMTE was about $8.82 \mathrm{~min}$. Urinary SBMTE levels of Patient 1 and 2 were measured as $314.43 \mathrm{ng} / \mathrm{mL}$ and $61.57 \mathrm{ng} / \mathrm{mL}$ respectively.

Conclusions:We confirmed SM exposure in urine samples of two patients by quantitative analysis of urinary SBMTE. The retrospective detection of urinary SBMTE supported SM exposure which was initially diagnosed with typical medical history, and presented typical symptoms.
\end{abstract}

\section{Introduction}

Sulfur mustard (CAS No. 505-60-2) which is defined as a "Schedule I chemical" in the Chemical Weapon Convention is a vesicant chemical warfare agent (CWA) (1). Sulfur mustard (SM) was first synthesized in 1822 by a Belgian Chemist, Cesar Despretz and the chemical formula was modified with a twostage synthesis process by a German Chemist, Victor Meyer in 1886 (2). SM was the first vesicant CWA which was used by Germans against Allied Forces in Ypres, Belgium on July 12, 1917 (3). Due to its devastating acute and chronic effects, SM was called as "King of Battle Gases" in World War I (2). SM was used in large scales by Iraq armed forces during the Iran-Iraq War between 1980 and 1988 (4). SM has a characteristics odor of mustard or horse radish and it is absorbed rapidly by inhalation, dermal contact, or from the anterior surface of the eye (5). It is a highly reactive compound which has carcinogenic and cytotoxic effects $(2,6)$. SM is also called as "radiomimetic drug" as effects of systemic SM poisoning are similar to chemotherapeutic agents (7). Except the literature which was related with chemical casualties of the Iran-Iraq War, chemical terrorist attacks against civilians in Middle East, accidental contact with old chemical munitions, and occupational accidents are the context of the recent literature about the clinical presentation of SM exposure $(8,9,10)$. The hallmark of the exposure is asymptomatic latency period which is typical for SM poisoning $(7,11)$.

The first non-specific symptoms of SM exposure in the first hour $(\mathrm{h})$ are mostly related with the eyes including conjunctivitis, soreness, and tearing. The respiratory tract and the skin are also affected (12). Except sore throat which is experienced rapidly due to inhalation of SM, symptoms including hoarseness, rhinorrhea, and coughing generally appear in 4-8 $\mathrm{h}$ after the exposure (10). Cutaneous symptoms of the exposure are erythema, vesicles, blisters, and deep layer burning in case of full epidermal loss. Intense itching generally occurs. The sequence of the cutaneous lesions are similarly seen in sunburn patients (11). Typical blister formation around joints especially in the knees and ankles lead to extreme pain which could reduce even simple movements (13). Neither erythema nor blister was noted on palms or soles due to the presence of thick layer of stratum corneum which protects the skin from chemicals (14).

The toxicity mechanism of SM exposure is still investigated 
and not fully elucidated. There are four major metabolic pathways of SM in humans $(8,15,16,17,18)$. First major pathway is hydrolysis of SM which leads to formation of thiodiglycol (TDG) and its oxidized form thiodiglycol sulfoxide (TDGO) (15). Second major pathway includes reaction of SM with glutathione and $\beta$-lyase cleavage of SM including 1,1'-sulfonylbis[2-(methylthio)ethane](SBMTE), 1-methylsulfinyl-2-[2-(methylthio)ethylsulfonyl] ethane (MSMTESE), and 1,1-sulfonylbis[2-(methyl-sulfinyl)ethane] (SBMSE) respectively $(17,19)$. Third major pathway is covalent binding of SM to nucleophiles in the bases of nucleic acids. Monoalkylation of guanine at N7 and O6, monoalkylation of adenine at N3, and bifunctional alkylation of guanine at N7 result in the formation of DNA adducts including N7-[2-[(2-hydroxyethyl) thio]ethyl]-guanine(N7-HETEG), O6-[2-[(2hydroxyethyl)thio]ethyl]-guanine (O6-HETEG), N3-[2-[(2-hydroxyethyl)thio]ethyl]-adenine (N3-HETEA), and bis[2-(guanin-7-yl)ethyl] sulfide (Bis-G) $(2,18)$. The fourth major pathway is binding of SM to Valine (Val) and Cysteine (Cys) residues of proteins which form adducts of hemoglobin (HETEVal) and albumin (HETE-Cys) (8, 20).

In our previous study, we reported typical symptomatology, clinical examination, and treatment modalities of a Syrian family who was exposed to SM during a chemical terrorist attack in North Syria in 2015. Gender and age of cases, their distance to the explosion are shown in Table I. The father, the mother, and two daughters were transferred from Marea -a city of North Syria- to Kilis State Hospital, Kilis on 18th h after the exposure. They presented typical ocular, respiratory, and cutaneous symptoms of SM poisoning at the time of admission. After a detailed medical examination, they were hospitalized with a diagnosis of chemical burn. Patient 1 and 2 were evacuated to an intensive care unit (ICU) in Gaziantep on 30th h after the exposure. Patient 3 and Patient 4 were evacuated to a pediatric ICU in Gaziantep on 48th h after the exposure (10).

\begin{tabular}{|c|c|c|c|}
\hline & Gender & Age & $\begin{array}{l}\text { Distance to the } \\
\text { explosion (meter) }\end{array}$ \\
\hline Patient 1 & Male & 31 & 3 \\
\hline Patient 2 & Female & 25 & 3 \\
\hline Patient 3 & Female & 3 & 3 \\
\hline Patient 4 & Female & 5-day-old & 3 \\
\hline
\end{tabular}

Our study aimed to confirm SM exposure by detecting quantitative levels of SBMTE in urine samples of a Syrian family that were victims of a chemical attack.

\section{Methods}

Four individuals who were exposed to SM were included into the study. Urine samples which were collected on 48th $\mathrm{h}$ upon admission to ICU's were analyzed for the retrospective detection of urinary SBMTE.

SBMTE (CAS No. 137371-98-3) was purchased from Spiez Laboratory, Switzerland. All solvents and reagents which were analytical grade were purchased from Sigma-Aldrich (Steinheim, Germany). Research-grade helium and argon gases were obtained from Ankara Gaz (Ankara, Turkey). Chromabond C8 (3 mL/500 mg) solid phase extraction (SPE) polypropylene cartridges were purchased from Macherey-Nagel (Düren, Germany). Blank urine samples were collected from volunteers.
An analytical method which was reported previously was used for the sample pretreatment and SPE (21). Urine sample $(500 \mu \mathrm{L})$ was mixed with $12 \% \mathrm{TiCl} 3$ solution $(250 \mu \mathrm{L})$ and the solution was heated at $75^{\circ} \mathrm{C}$ for 60 minutes (min) for the complete reduction of MSMTESE and SBMSE to SBMTE. After neutralization and precipitation, the supernatant was passed through a C8 SPE cartridge $(3 \mathrm{~mL} / 500 \mathrm{mg}$ ) which was preconditioned with methanol $(1000 \mu \mathrm{L})$ and distilled water $(1000 \mu \mathrm{L})$ under vacuum. Following rinsing interferences with methanol/ distilled water $(1000 \mu \mathrm{L})$, purified analyte was eluted with methanol $(1000 \mu \mathrm{L})$ into a $\mathrm{GC}$ vial $(2 \mathrm{~mL})$.

Shimadzu GCMS-TQ8040 (Tokyo, Japan) gas chromatography-tandem mass spectrometry (GC-MS-MS) which was equipped with a $30-\mathrm{m}$ Rxi-5ms capillary column $(0.25 \mathrm{~mm}$ i.d. and $0.25 \mu \mathrm{m}$ film thickness, Restek, Bellefonte, USA) and a $5 \mathrm{~m}$ Rxi guard column ( 0.25 mm i.d., Restek, Bellefonte, USA) was used for the instrumental analysis of urinary $\beta$-lyase metabolite SBMTE. Reported parameters were used for the instrumentation (21). The temperature program of GC oven started at $40^{\circ}$ $\mathrm{C}(1 \mathrm{~min})$, it was heated by a linear ramp of $30^{\circ} \mathrm{C} / \mathrm{min}$ to $280^{\circ} \mathrm{C}$ and kept at $280^{\circ} \mathrm{C}$ for $4 \mathrm{~min}$. A final concentration of $1 \mathrm{mg} / 1 \mathrm{~mL}$ SBMTE standard was obtained by dissolving SBMTE $(5 \mathrm{mg})$ in methanol $(5 \mathrm{~mL})$. Quantitative analysis of urinary SBMTE was performed by using external calibration standards $(2.5,5,10$, 25,50 , and $100 \mathrm{ng} / \mathrm{mL}$ SBMTE) in the multiple reaction monitoring (MRM) mode. A linear calibration curve was obtained over the range $2.5-100 \mathrm{ng} / \mathrm{mL}$ ( $r 2>0.99)$. The quantitative ions of SBMTE was $140 \rightarrow 75 \mathrm{~m} / \mathrm{z}$.

The present study was approved by the Ethical Board of Gulhane Training and Research Hospital, Ankara, Turkey.

\section{Results}

Typical symptoms of SM exposure including bilateral conjunctivitis, blepharospasm, photophobia, sore throat, productive coughing, rhinorrhea, severe itching, diffuse erythema, and numerous multiple blisters with various sizes. These symptoms were observed in our patients on 18th hour after the exposure as shown in Figure 1 and 2. Urine samples were collected on 48th $\mathrm{h}$ after the exposure for further diagnosis and these samples were analyzed by using an analytical method that was

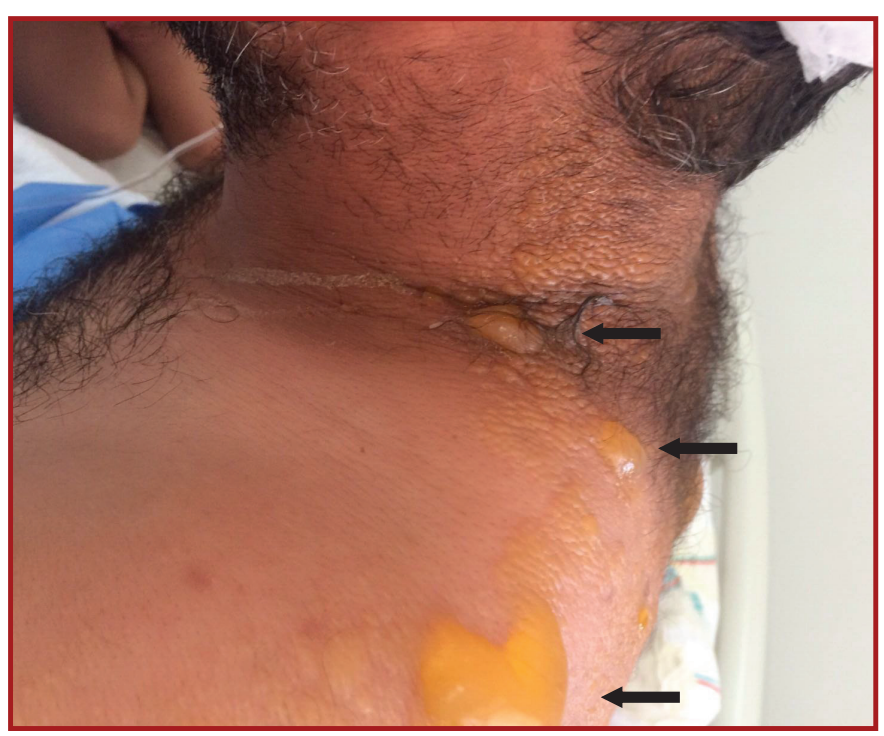

Figure 1. Typical blister formations due to SM contact on the left shoulder of Patient 1 on 18th $\mathrm{h}$ after the exposure. 


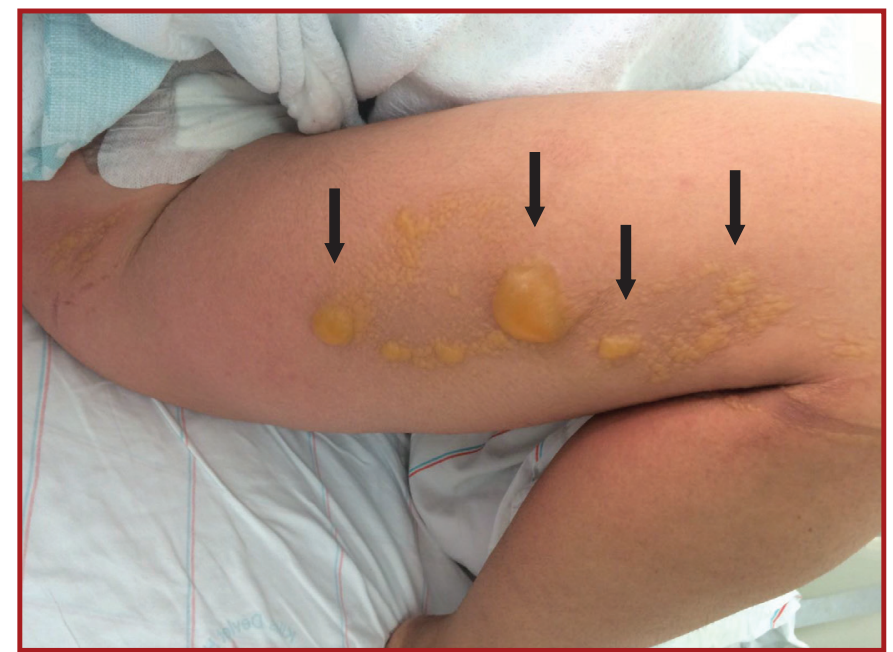

Figure 2. Typical blister formations due to SM contact on the right lower extremity of Patient 2 on 18th h after the exposure.

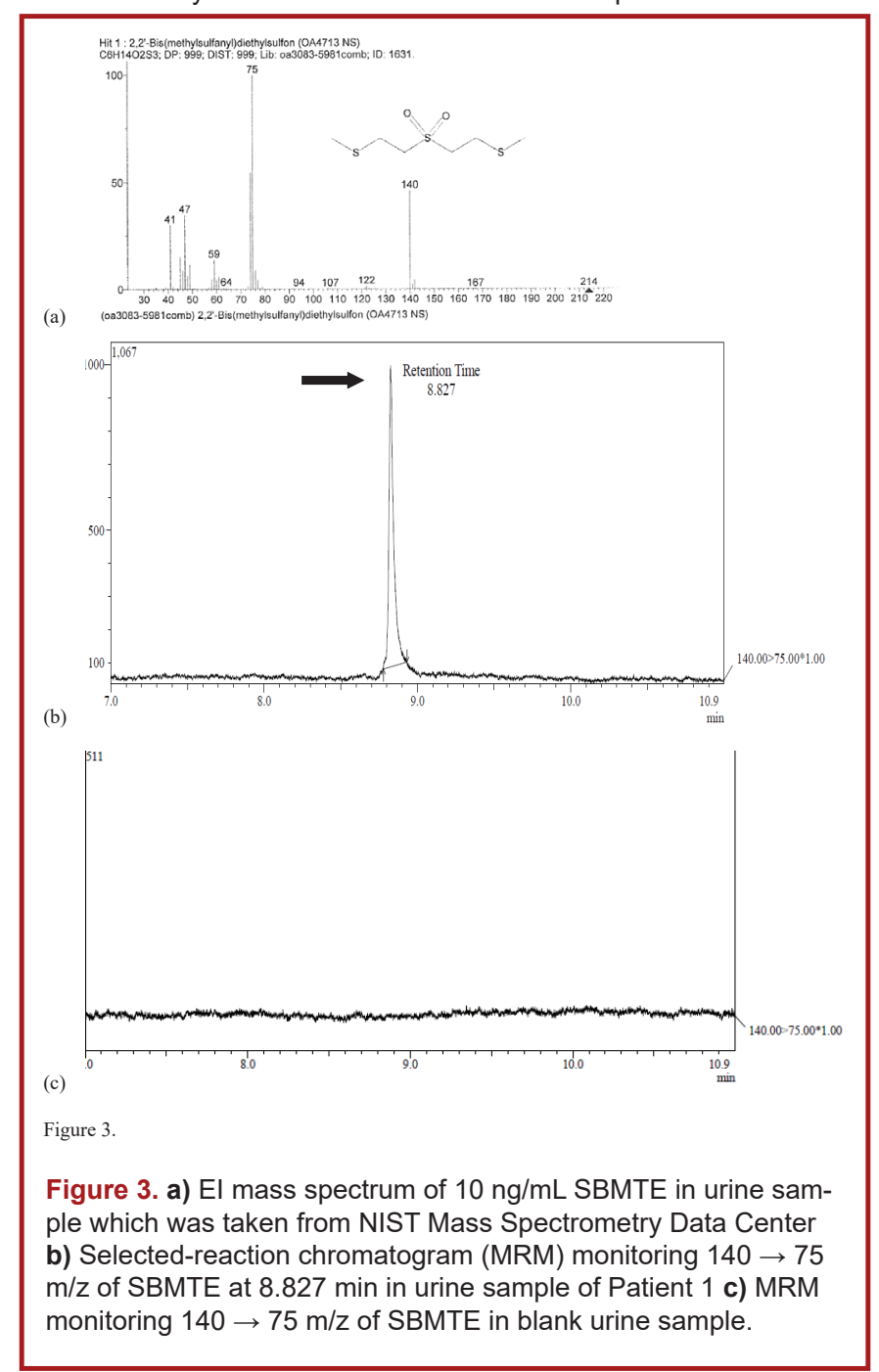

modified for the quantitation of urinary SBMTE (21). The limit of detection (LOD) for SBMTE was $1 \mathrm{ng} / \mathrm{mL}$ and the retention time for SBMTE was about $8.82 \mathrm{~min}$ (Figure 3).

Urinary SBMTE levels of Patient 1 and 2 were measured as $314.43 \mathrm{ng} / \mathrm{mL}$ and $61.57 \mathrm{ng} / \mathrm{mL}$ respectively. There was no SBMTE measured in urine sample of Patient 3. SBMTE was not determined due to insufficient urine sample of Patient 4.

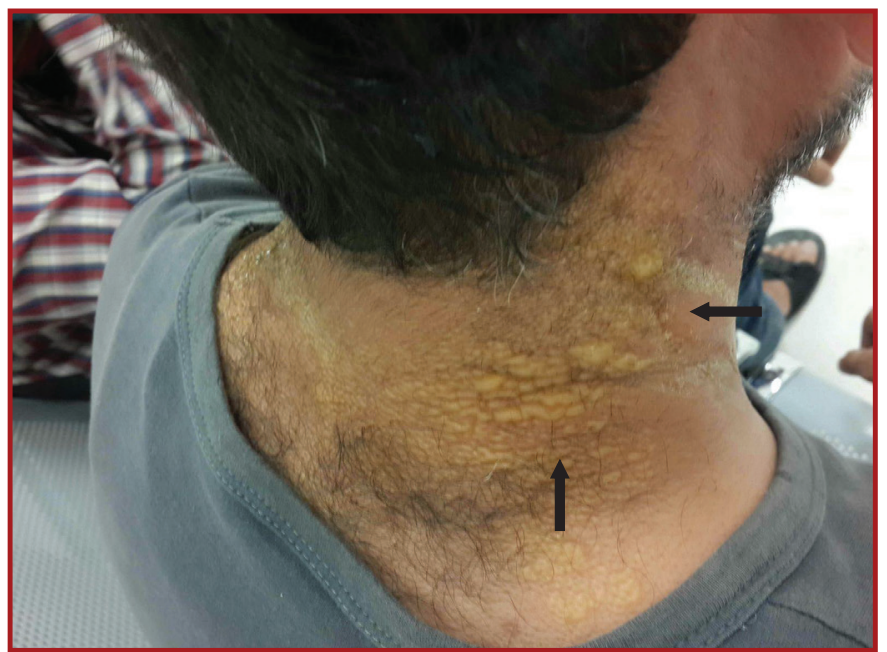

Figure 4. Typical blister formations due to SM contact on the neck of Patient 1 on 18th $\mathrm{h}$ after the exposure.

\section{Discussion}

Our cases were exposed to a blister agent during a chemical terrorist attack. They presented typical delayed onset of symptoms and the first ocular symptoms occurred on 10 to 30 min after the exposure. Blister formations as shown in Figure 4 were observed on 18th $\mathrm{h}$ after the exposure (10). The onset and severity of symptoms depend on concentration of SM in the air, ambient humidity and temperature of the scene, and existence or absence of personal protective equipment during the exposure (13). As our cases were civilian, they had no personal protective equipment against CWA's during the chemical attack and they were only few meters away from the explosion. For this reason, we concluded that they had severe second-degree burn lesions especially in mean of severity and extensity as they were exposed directly to mustard gas in high concentrations.

As conjunctivitis, corneal swelling, and edema occur with doses exceeding $200 \mathrm{mg} \cdot \mathrm{min} / \mathrm{m} 3$ and blister formation occurs at higher doses (vapor: 1000-2000 mg. $\mathrm{min} / \mathrm{m}^{3}$, liquid), depending on presented symptoms, we concluded that the whole family $(n=4)$ was exposed to SM at a dose higher than 1000 $\mathrm{mg} \cdot \mathrm{min} / \mathrm{m}^{3}(5,12)$.

Cutaneous symptoms of SM including erythema, blisters, and even necrosis that accompany red eye and coughing are similar with symptoms of either thermal or chemical burns (22). For this reason, further bioanalytical diagnosis should be performed upon admission of chemical casualties into the emergency department. Retrospective verification of SM exposure even if at low levels is vital in mean of expecting the level of the absorbed dose due to the exposure $(1,22)$. Additionally, bioanalysis of samples which were belonged to chemical casualties for the detection of both metabolites and covalent adducts could support forensic verification of the exposure in mean of evidence of alleged use of CWA's (23). Besides $\beta$-lyase metabolites, DNA and protein adducts could be also used for the verification of SM exposure as they could be found in urine samples up to 30 days and they could persist several weeks in human blood after the exposure $(8,17)$.

Human biological samples including blood and urine are the most accessible specimens early after chemical terrorist attacks especially during the initial medical management of chemical casualties at the emergency department. As timing is 
very important for the forensic sampling, it should be noted that necessary measures should be taken in order to collect biological specimens including blood and urine from the casualties as soon as possible. After the sampling, all biological evidences which are divided into aliquots should be transferred in ideal storage conditions to the reference off-site laboratories which have such analytical capabilities.

There are some studies in the literature which quantitated $\beta$-lyase metabolites of SM, especially urinary SBMTE in victims of SM exposure because $\beta$-lyase metabolites are unequivocal diagnostic biomarkers for SM exposure $(9,15,19,24)$. We also preferred analyzing urinary SBMTE for the retrospective detection of SM exposure in our cases. Our modified method depended on reducing polar ß-lyase metabolites MSMTESE and SBMSE by using titanium trichloride to low polar ß-lyase metabolite SBMTE which had more suitable chemical structure for GC-MS-MS analysis $(1,21)$.

Our study confirmed that urine samples of Patient 1 and 2 had detectable levels of SBMTE. Level of SBMTE could not be measured in urine sample of Patient 3 despite the existence of typical symptoms of SM poisoning so we concluded that the level of SBMTE in urine sample of Patient 3 could be lower than our method's LOD. We could not analysis urine sample of Patient 4 as her urine sample was not stored in proper conditions. Thus, we concluded that the specimen collection method and the physical conditions of the specimen in mean of proper storing containers were also critical factors in order to obtain proper specimen's aftermath of a chemical terrorist attack.

Studies that confirmed SM exposure in victims of chemical attacks are especially rare and samples were only belonged to veterans of the Iran-Iraq War $(15,25)$. Our study also confirmed SM exposure by chromatographic methods in urine samples of victims of chemical terrorism. It was assumed that there was a correlation between levels of SBMTE in urine samples and severity of SM poisoning (22). We measured high level of SBMTE in urine samples of Patient 1 and 2 who were severe ocular, respiratory, and cutaneous lesions. We concluded that our findings also showed this correlation.

It is hard enough to transport ready-made chemical weapons over long distances, but non-state actors could use microsynthesis methods in order to prepare CWA's in the target countries early before such a chemical terrorist attack. For this reason, alleged use of chemical warfare agents especially SM against civilians could be a part of chemical terrorist attack. Such a chemical warfare agent like SM with a symptom-free latency period after the exposure, it could be problematic to differ worried-well patients from real victims. Although detailed medical examination of related symptoms could be enough for early diagnosis of SM exposure, analytical capabilities for the retrospective verification of SM exposure should be performed in off-site laboratories as soon as possible with suitable biological samples which were belonged to victims of the chemical attack.

\section{Acknowledgments}

Authorship contributions: Concept: S.S.; Design: S.S, R.K.E.; Supervision: L.K; Resources: S.S, R.K.E.; Materials: S.S, R.K.E.; Data collection and/or processing: S.S, R.K.E.; Analysis and/or interpretation: S.S, R.K.E, L.K.; Writing: S.S.; Critical review: L.K.

\section{Conflict of Interest}

The authors declared they do not have anything to disclose regarding conflict of interest with respect to this manuscript.

\section{References}

1. Young CL, Ash D, Driskell WJ, et al. A rapid, sensitive method for the quantitation of specific metabolites of sulfur mustard in human urine using isotope-dilution gas chromatography-tandem mass spectrometry. J Anal Toxicol. 2004;28(5):339-345.

2. Balali-Mood M, Abdollahi M. Basic and clinical toxicology of mustard compounds. 1st ed. New York: Springer; 2015.

3. Romano Jr. JA, Salem H, Lukey BJ. Chemical warfare agents: chemistry, pharmacology, toxicology, and therapeutics. 1st ed. CRC Press; 2007.

4. Kilic E, Ortatatli M, Sezigen S, Eyison RK, Kenar L. Acute intensive care unit management of mustard gas victims: the Turkish experience. Cutan Ocul Toxicol. 2018;37(4):332-337.

5. Balali-Mood M, Hefazi M. The pharmacology, toxicology, and medical treatment of sulphur mustard poisoning. Fundam Clin Pharmacol. 2005;19(3):297-315.

6. Sezigen S, Karayılanoğlu T. Kimyasal savaş ajanlarının solunum sistemine etkileri ve tedavi yaklaşımları. Turk Hij Den Biyol Derg. 2006;63(1):129-134.

7. Kehe K, Szinicz L. Medical aspects of sulphur mustard poisoning. Toxicology. 2005;214(3):198-209.

8. Xu H, Nie Z, Zhang Y, Li C, et al. Four sulfur mustard exposure cases: Overall analysis of four types of biomarkers in clinical samples provides positive implication for early diagnosis and treatment monitoring. Toxicol Rep. 2014;1:533-543.

9. Steinritz D, Striepling E, Rudolf KD, et al. Medical documentation, bioanalytical evidence of an accidental human exposure to sulfur mustard and general therapy recommendations. Toxicol Lett. 2016;244:112-120.

10. Sezigen S, Ivelik K, Ortatatli M, et al. Victims of chemical terrorism, a family of four who were exposed to sulfur mustard. Toxicol Lett. 2019;303:9-15.

11. Balali-Mood, M, Mathews R, Pita R, et al. Practical guide for medical management of chemical warfare casualties. http://sbu.edu.tr/FileFolder/Dosyalar/eb408a43/2018_6/ kimyasal_savas_yaralilarinin.pdf. Accessed February 08,2018

12. Kehe K, Thiermann H, Balszuweit F, Eyer F, Steinritz D, Zilker T. Acute effects of sulfur mustard injury-Munich experiences. Toxicology. 2009;263(1):3-8.

13. Otter J, Dawood A, D'Orazio J. Sulfur mustard exposure from dredged artillery shell in a commercial clammer. Clin Pract Cases Emerg Med. 2017;1(4):283-286.

14. Malhotra RC, Ganesan K, Sugendran K, Swamy RV. Chemistry and toxicology of sulphur mustard-a review. Defence Science Journal. 1999;49(2):97-116.

15. Black RM, Read RW. Improved methodology for the de- 
tection and quantitation of urinary metabolites of sulfur mustard using gas chromatography-tandem mass-spectrometry. J Chromatogr B Biomed Appl. 1995;665(1):97105.

16. LinY, DongY, ChenJ, et al. Gas chromatographic-tandem mass spectrometric analysis of $\beta$-lyase metabolites of sulfur mustard adducts with glutathione in urine and its use in a rabbit cutaneous exposure model. J Chromatogr B Analyt Technol Biomed Life Sci. 2014;945-946:233239.

17. Daly JD, O'Hehir CM, Frame GM. A sensitive method for quantitation of beta-lyase metabolites of sulfur mustard as 1,1'-sulfonylbis[2-(methylthio)ethane] (SBMTE) in human urine by isotope dilution liquid chromatography-positive ion-electrospray-tandem mass spectrometry. J Chromatogr B Analyt Technol Biomed Life Sci. 2007;850(1-2):120-127.

18. Zubel T, Bürkle A, Mangerich A. Mass spectrometric analysis of sulfur mustard-induced biomolecular adducts: Are DNA adducts suitable biomarkers of exposure? Toxicol Lett. 2018;293:21-30.

19. Barr JR, Pierce CL, Smith JR, et al. Analysis of urinary metabolites of sulfur mustard in two individuals after accidental exposure. J Anal Toxicol. 2008;32(1):10-16.

20. Pantazides BG, Crow BS, Garton JW, et al. Simplified method for quantifying sulfur mustard adducts to blood proteins by ultrahigh pressure liquid chromatographyisotope dilution tandem mass spectrometry. Chem Res Toxicol. 2015;28(2):256-261.

21. Eyison RK, Sezigen S, Ortatatli M, et al. Optimized gas chromatography-tandem mass spectrometry for 1,1'-sulfonylbis[2-(methylthio) ethane] quantification in human urine. J Chromatogr Sci. 2019. doi: 10.1093/chromsci/ bmz017.

22. Lin Y, Dong Y, Chen J, Li CZ, Nie ZY, Guo L, Liu Q, Xie JW. Gas chromatographic-tandem mass spectrometric analysis of $\beta$-lyase metabolites of sulfur mustard adducts with glutathione in urine and its use in a rabbit cutaneous exposure model. J Chromatogr B Analyt Technol Biomed Life Sci. 2014;945-946:233-239.

23. Black RM. An overview of biological markers of exposure to chemical warfare agents. J Anal Toxicol. 2008;32(1):29.

24. Read RW, Black RM. Analysis of beta-lyase metabolites of sulfur mustard in urine by electrospray liquid chromatography-tandem mass spectrometry. J Anal Toxicol. 2004;28(5):346-351.

25. Willems JL. Clinical management of mustard gas casualties. J. Anna Med. Militaris. 1989;3:1-61. 